Pacific Journal of Mathematics

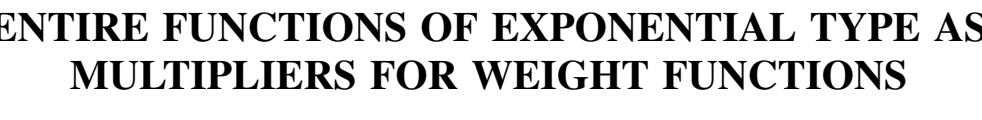




\title{
ENTIRE FUNCTIONS OF EXPONENTIAL TYPE AS MULTIPLIERS FOR WEIGHT FUNCTIONS
}

\author{
PAUL Koosis
}

Dédié à la mémoire de ma mère, Frieda Smith, née Levin, 3 novembre 1907 - 22 novembre 1979.

If $W(x) \geqq 1$ is defined on the real line and satisfies (1), a discussion is given of the regularity assumptions which must be imposed on $W$ in order to guarantee the existence of nonzero entire functions $\varphi$ of arbitrarily small exponential type making $W(x) \varphi(x)$ bounded on the real axis. It is known that such $\varphi$ exist provided that $\log W(x)$ is uniformly Lip 1 . An example is given which shows, among other things, that this is no longer the case if we merely suppose that $\log \log W(x)$ is uniformly Lip 1 .

1. Introduction. Suppose we are given a function $W(x) \geqq 1$ (henceforth called a weight), defined for $-\infty<x<\infty$ and satisfying

$$
\int_{-\infty}^{\infty} \frac{\log W(x)}{1+x^{2}} d x<\infty
$$

In connection with various problems, it is important to know when there exist nonzero entire functions $\varphi(z)$ of exponential type for which $W(x) \varphi(x)$ is bounded, or belongs at least to some $L_{p}$ class, on the real axis. Some of these problems involve the completeness of sets of exponentials on finite intervals ([12], p. 50ff, [1]) or other aspects of approximation theory ([4], pp. 254-288). Let us also mention the question of nonuniqueness for the best $H_{\infty}$-approximations to certain functions of unit modulus related to $W([5], \S 4)$, and a closely related one concerning the existence of nonzero positive functions $\omega(x)$ such that

$$
\int_{-\infty}^{\infty}|\widetilde{f}(x)|^{2} \omega(x) d x \leqq \int_{-\infty}^{\infty} \frac{|f(x)|^{2}}{W(x)} d x
$$

for the Hilbert transform $\tilde{f}$ of each bounded function $f$ having its spectrum in $(-\infty,-a] \cup[a, \infty), a>0$ ([5], [6]).

If, besides (1), $W(x)$ satisfies certain regularily conditions, there are nonzero entire functions $\phi$ of arbitrarily small exponential type making $W(x) \varphi(x)$ bounded for real $x$. It has long been known, for instance, that this is the case if $W(x)$ is even and increasing for $x>0$ (see, inter alia, [10], p. 81, [7], §5); the theorem on the multiplier, due to Beurling and Malliavin, furnishes the same result 
whenever $W$, satisfying (1), is itself an entire function of exponential type, or has a logarithm uniformly continuous on the real axis ([2], [8], [11]). The mere relation (1) is certainly not sufficient by itself for the existence of such $\varphi$, and it seems very difficult to get a simple description of minimal regularity conditions whose imposition on $W(x)$ would ensure that existence.

For this reason the following question seems natural:

If there is some nonzero entire function $\Phi$ of exponential type, with (for instance)

$$
\int_{-\infty}^{\infty}|\Phi(x)| W(x) d x<\infty,
$$

are there nonzero entive functions $\varphi$ of arbitrarily small exponential type such that

$$
\int_{-\infty}^{\infty}|\varphi(x)| W(x) d x<\infty ?
$$

The simplest examples show that even here $W(x)$ must have some regularity in order for there to be a positive answer. Take, for instance,

$$
W(x)=\left(\frac{\pi x}{\sin \pi x}\right)^{2}
$$

then (2) holds with

$$
\Phi(x)=\left(\frac{\sin \pi x \sin \eta x}{x^{2}}\right)^{2},
$$

an entire function of exponential type $2 \pi+2 \eta$. Any entire function $\varphi$ satisfying (3) must, however, have a double zero at least at each of the points $\pm 1, \pm 2, \pm 3, \cdots$, and this, by Carlson's theorem ([3], p. 153), cannot happen for a nonzero $\varphi$ of exponential type $<2 \pi$. This example, which works because $W(n)=\infty$ at the nonzero integers $n$, seems to have an artificial character; it would be excluded from our discussion by requiring $W$ to satisfy an inequality like

$$
W\left(x^{\prime}\right) \geqq(W(x))^{c}
$$

whenever $\left|x^{\prime}-x\right| \leqq l$, with two positive constants $c$ and $l$. This is a kind of uniform semi-continuity, much weaker than the uniform continuity of $\log W(x)$ which, as noted above, is already enough to ensure existence for nonzero $\phi$ of arbitrarily small exponential type satisfying (3).

Let us agree to henceforth only consider weights $W(x)$ having at least the following regularity: 
There are two positive constants $c$ and $l$ such that, for each $x_{0}$, we have

$$
W(x) \geqq\left(W\left(x_{0}\right)\right)^{c}
$$

throughout some interval of length $l$ containing $x_{0}$.

This condition, milder than the one mentioned previously, holds whenever, starting from any function $\Omega(x) \geqq 1$ satisfying

$$
\int_{-\infty}^{\infty} \frac{\log \Omega(x)}{1+x^{2}} d x<\infty,
$$

we form the geometrically smoothed weight

$$
W(x)=\exp \left\{\frac{1}{2 l} \int_{-l}^{l} \log \Omega(x+t) d t\right\} .
$$

Indeed, for any $x$, at least one of the two relations

$$
\begin{aligned}
& \frac{1}{2 l} \int_{-l}^{0} \log \Omega(x+t) d t \geqq \frac{1}{2} \log W(x), \\
& \frac{1}{2 l} \int_{0}^{l} \log \Omega(x+t) d t \geqq \frac{1}{2} \log W(x)
\end{aligned}
$$

must hold. So, since $\log \Omega \geqq 0$, we have $W\left(x^{\prime}\right) \geqq \sqrt{W(x)}$ on at least one of the two intervals $x-l \leqq x^{\prime} \leqq x, x \leqq x^{\prime} \leqq x+l$, giving us (4) with $c=1 / 2$.

If condition (4) on $W$ is fulfilled and there exists any nonzero $\Phi$ of exponential type satisfying (2), we can find another entire $\Psi$ of exponential type (perhaps larger than that of $\Phi$ ), having only real zeros, and such that

$$
\int_{-\infty}^{\infty}|\Psi(x+i)| W(x) d x<\infty
$$

To see this, start with the Hadamard product representation

$$
\Phi(z)=A z^{p} e^{i \alpha z} \prod_{\lambda}\left(1-\frac{z}{\lambda}\right) e^{z / \lambda} ;
$$

here, $\lambda$ runs through the zeros $\Phi$ has away from the origin, and $p$ is a nonnegative integer. Since $W \geqq 1$, (2) implies $\int_{-\infty}^{\infty}|\Phi(x)| d x<\infty$, so $|\Phi(x)|$ is bounded for real $x$ ([3], p. 98). This makes the constant $\alpha$ in (7) real. In the right-hand side of (7), let us now throw away all factors corresponding to purely imaginary zeros $\lambda$, and replace each remaining $\lambda$ by a real one, $\tilde{\lambda}$, related to it through the formula

$$
1 / \widetilde{\lambda}=\Re(1 / \lambda) \text {. }
$$


This procedure gives us a new entire function $\Phi_{0}(z)$ of exponential type which is easily seen to satisfy the inequality $\left|\Phi_{0}(x)\right| \leqq|\Phi(x)|$ for real $x$, and hence also

$$
\int_{-\infty}^{\infty}\left|\Phi_{0}(x)\right| W(x) d x<\infty,
$$

(According to [10], p. 30, the type of $\Phi_{0}$ is less than or equal to that of $\Phi$; here, the boundedness of $\Phi$ on the real axis makes the two types equal. See [12], pp. 55-56 and [3], p. 115.)

Calling $A$ the type of $\Phi_{0}$ in the upper half plane, we have, by Poisson's formula,

$$
\log \left|\Phi_{0}(x+i)\right|=A+\frac{1}{\pi} \int_{-\infty}^{\infty} \frac{\log \left|\Phi_{0}(t)\right| d t}{(x-t)^{2}+1},
$$

because $\Phi_{0}$ has only real zeros ([3], p. 92). Jensen's inequality, combined with (8), now yields

$$
\begin{array}{r}
\log \left|\Phi_{0}(x+i)\right|+\frac{1}{\pi} \int_{-\infty}^{\infty} \frac{\log W(x+t)}{t^{2}+1} d t \\
\leqq A+\log \left\{\frac{1}{\pi} \int_{-\infty}^{\infty} \frac{\left|\Phi_{0}(t)\right| W(t) d t}{(x-t)^{2}+1}\right\} \\
\leqq A+\log \left\{\frac{1}{\pi} \int_{-\infty}^{\infty}\left|\Phi_{0}(t)\right| W(t) d t\right\},
\end{array}
$$

so

$$
\left|\Phi_{0}(x+i)\right| \exp \left\{\frac{1}{\pi} \int_{-\infty}^{\infty} \frac{\log W(x+t)}{t^{2}+1} d t\right\}
$$

is bounded for $-\infty<x<\infty$.

Condition (4), however, yields

$$
\frac{1}{\pi} \int_{-\infty}^{\infty} \frac{\log W(x+t)}{t^{2}+1} d t \geqq \frac{c l \log W(x)}{\pi\left(l^{2}+1\right)},
$$

so $\left|\Phi_{0}(x+i)\right|(W(x))^{k} \leqq K$ on the real axis with two positive constants $k$ and $K$. Since $W(x) \geqq 1$, we see that $\left|\Phi_{0}(x+i)\right|{ }^{N} W(x)$ is bounded for real $x$ if $N$ is any integer $>1 / k$, and the formula

$$
\Psi(z)=\frac{\sin ^{2} z}{z^{2}}\left(\Phi_{0}(z)\right)^{N}
$$

now provides us with a nonzero entire function $\Psi$ of exponential type having only real zeros and satisfying (6).

Suppose that we merely have some function $\Omega(x) \geqq 1$ and a nonzero $\Phi$ of exponential type such that

$$
\int_{-\infty}^{\infty}|\Phi(x)| \Omega(x) d x<\infty .
$$


An argument like the preceding one can then be carried through with $\Omega$ instead of $W$, up to the point where an inequality

$$
\left|\Phi_{0}(x+i)\right| \exp \left\{\frac{1}{\pi} \int_{-\infty}^{\infty} \frac{\log \Omega(x+t)}{t^{2}+1} d t\right\}<K,-\infty<x<\infty,
$$

is obtained. However, if $W$ is the smoothed weight obtained from $\Omega$ by (5), we have

$$
\frac{1}{\pi} \int_{-\infty}^{\infty} \frac{\log \Omega(x+t)}{t^{2}+1} d t \geqq \frac{2 l \log W(x)}{\pi\left(l^{2}+1\right)},
$$

and we see that (6) still holds with a suitable entire $\Psi$ having only real zeros.

On account of (6), the existence of entire $\varphi$ of arbitrarily small exponential type satisfying (3) is seen to boil down to existence of such $\varphi$ for which

$$
\frac{|\varphi(x)|}{|\Psi(x+i)|} \leqq \text { const. },-\infty<x<\infty .
$$

Taken generally, this statement has a converse:

If, for every weight $W$ fulfilling (4), the existence of one entire $\Phi$ of exponential type satisfying (2) implies that of entire $\varphi$ with arbitrarily small type satisfying (3), then, for every entire $\Psi$ bounded on the real axis and having all its zeros there, we do have entire $\varphi$ of arbitrarily small type satisfying (9).

To see this, let $\Psi(z)$, having only real zeros, be of exponential type $A$ in $\Im z>0$ and satisfy $|\Psi(x)| \leqq 1,-\infty<x<\infty$. A PhragménLindelöf theorem ([3], p. 82) makes $|\Psi(x+i / 2)| \leqq e^{A / 2}$, and we can take the weight

$$
W(x)=\frac{e^{A / 2}}{|\Psi(x+i / 2)|} .
$$

A relation similar to (8),

$$
\log |\Psi(x+i / 2)|=\frac{1}{2} A+\frac{2}{\pi} \int_{-\infty}^{\infty} \frac{\log |\Psi(t)| d t}{4(x-t)^{2}+1},
$$

yields, on differentiation,

$$
\left|\frac{d \log |\Psi(x+i / 2)|}{d x}\right| \leqq 2 \log \left|\frac{e^{A / 2}}{\Psi(x+i / 2)}\right|,
$$

because $\log |\Psi(t)| \leqq 0$. Our $W$ thus satisfies (4) with, say, $l=1$ and $c=1 / e^{2}$.

There is certainly a nonzero entire $\Phi$ of exponential type satisfying (2), for instance, $\Phi(z)=\left(\sin ^{2} z / z^{2}\right) \Psi(z+i / 2)$. Suppose now that 
$\phi$ is any entire function, of exponential type equal, say, to $a$. Then ([3], p. 93),

$$
\log |\varphi(x+i / 2)| \leqq \frac{a}{2}+\frac{2}{\pi} \int_{-\infty}^{\infty} \frac{\log |\varphi(t)| d t}{4(x-t)^{2}+1},
$$

so, since

$$
\log |\Psi(x+i)|=\frac{A}{2}+\frac{2}{\pi} \int_{-\infty}^{\infty} \frac{\log |\Psi(t+i / 2)|}{4(x-t)^{2}+1} d t,
$$

we have, by Jensen's inequality,

$$
\log \left|\frac{\varphi(x+i / 2)}{\Psi(x+i)}\right| \leqq \frac{a}{2}-A+\log \frac{2}{\pi}\left\{\int_{-\infty}^{\infty} \frac{|\varphi(t)| W(t)}{4(x-t)^{2}+1} d t\right\},
$$

in terms of our weight $W$. If, now, $\varphi$ satisfies (3), we see that (9) holds with $\varphi(x+i / 2)$ standing in place of $\varphi(x)$.

\section{Truth of the}

AFFirmation. For every nonzero $\Psi$ of exponential type bounded on the real axis and having all its zeros there, there exist nonzero entire $\phi$ of arbitrarily small exponential type such that $\varphi(x) / \Psi(x+i)$ is bounded for real $x$

is thus both necessary and sufficient for the validity of the following

Proposition. For weights $W$ having the regularity property (4), existence, for any particular one of them, of a nonzero entive $\Phi$ of exponential type satisfying (2) implies that of nonzero entire $\varnothing$ with arbitrarily small type satisfying (3).

This makes proof or disproof of the affirmation rather important, in spite of the special nature of the factor $1 / \Psi(x+i)$ appearing in its formulation. In the following section an example is constructed which shows the affirmation to be false. Therefore the proposition is also false.

2. The example. Starting with some large value of $p$, say $p=8$, let us put

$$
x_{p}=\exp p^{1 / 3} .
$$

Take $\Delta_{8}=x_{3}$, and, for $p>8$, write

$$
\Delta_{p}=x_{p}-x_{p-1} .
$$


We form the even function

$$
F(z)=\prod_{p \geq 8}\left(1-\frac{z^{2}}{x_{p}^{2}}\right)^{\left[s_{p}\right]}
$$

( $\left[\Delta_{p}\right]$ means the greatest integer $\left.\leqq \Delta_{p}\right)$; convergence of the infinite product on the right is easily verified, and $F(z)$ is an entire function having a zero of multiplicity $\left[\Delta_{p}\right]$ at each point $\pm x_{p}, p \geqq 8$.

For $t>0$, denote by $n(t)$ the number of zeros of $F(z)$ (counting multiplicities) lying between 0 and $t$. If $p>\infty$ and $t$ lies between $x_{p}$ and $x_{p+1}$,

$$
n(t)=\left[\Delta_{8}\right]+\cdots+\left[\Delta_{p}\right]
$$

lies between $x_{p}-p$ and $x_{p}$, where, by $(10), p=\left(\log x_{p}\right)^{3}$. Since $x_{p+1} / x_{p} \underset{p}{ } 1$, we clearly have

$$
\frac{n(t)}{t} \longrightarrow 1, t \longrightarrow \infty,
$$

and this implies, as is well known ([3], p. 136), that $F(z)$ is of exponential type $\pi$.

Given any $\eta>0$ (which we now choose and fix once and for all), let us show that there is a nonzero even entire function $g(z)$ of exponential type $\leqq \eta$ having only real zeros with $F(z) g(z)$ bounded on the real axis. This we can do by appealing to the old result (about even weights $W(x)$ increasing on the positive real axis) mentioned near the beginning of the introduction. For $x_{p} \leqq t<x_{p+1}$, we have $t \geqq x_{p} \geqq n(t) \geqq x_{p}-p \geqq t-p-\Delta_{p+1}$. By (10) and (11), for large $p$,

$$
\Delta_{p} \sim \Delta_{p+1} \sim \frac{1}{3} p^{-2 / 3} x_{p}=\frac{1}{3} \frac{x_{p}}{\left(\log x_{p}\right)^{2}},
$$

so, if $x_{p} \leqq t<x_{p+1}$,

$$
t-\left(\frac{1}{3}+o(1)\right) \frac{x_{p}}{\left(\log x_{p}\right)^{2}}-\left(\log x_{p}\right)^{3} \leqq n(t) \leqq t,
$$

where the term $o(1)$ tends to zero as $p \rightarrow \infty$. We can thus surely write

$$
t-\frac{t}{(\log t)^{2}} \leqq n(t) \leqq t
$$

for all sufficiently large values of $t(n(t) \leqq t$ for all $t>0)$.

The above definition of the function $F$ can be rewritten as $\log |F(x)|=\int_{0}^{\infty} \log \left|1-x^{2} / t^{2}\right| d n(t)$; after integrating by parts and chang- 
ing variables ([7], pp. 127-128), this becomes, for $x>0$,

$$
\log |F(x)|=2 \int_{0}^{1} \frac{\frac{n(x \tau)}{\tau}-\tau n\left(\frac{x}{\tau}\right)}{1-\tau^{2}} d \tau .
$$

Since $n(t)$ is increasing, the integral on the right is

$$
\leqq 2 n(x) \log \frac{1}{\lambda}+2 \int_{0}^{\lambda} \frac{\frac{n(x \tau)}{\tau}-\tau n\left(\frac{x}{\tau}\right)}{1-\tau^{2}} d \tau
$$

for any positive number $\lambda$ less than 1 . Substituting (13) into this expression, we find

$$
\log |F(x)| \leqq 2 x \log \frac{1}{\lambda}+2 x \int_{0}^{\lambda} \frac{d \tau}{[\log (x / \tau)]^{2}\left(1-\tau^{2}\right)}
$$

and, taking $\lambda=1-1 /(\log x)^{2}$, we get

$$
\log |F(x)| \leqq \frac{C x \log \log x}{(\log x)^{2}}
$$

for large $x, C$ being a constant. (The same result may also be obtained directly by just plugging $H(x)=x /(\log x)^{2}$ into Theorem 51, p. 35 of [12].)

The right hand member of (14) is increasing for large $x$, and satisfies

$$
\int_{e}^{\infty} \frac{C x \log \log x}{(\log x)^{2}} \frac{d x}{x^{2}}<\infty .
$$

An old theorem (see [10], p. 81, [7], §5, or [12], pp. 26-27-it is not necessary to resort to the Beurling-Malliavin multiplier theorem here) now furnishes us with a nonzero even entire function $g(z)$ having only real zeros and of exponential type $\leqq \eta$, such that $F(x) g(x)$ is bounded for real $x$; putting

$$
\Psi(z)=F(z) g(z),
$$

we have a nonzero even entire function $\Psi$ of exponential type $\leqq \pi+\eta$, bounded on the real axis and having only real zeros, among which are the $\left[\Delta_{p}\right]$-fold ones at the points $\pm x_{p}$.

$I$ say now that there can be no nonzero entire function $\varphi$ of exponential type $a<\pi$ for which $|\varphi(x) / \Psi(x+i)|$ is bounded on the real axis. Suppose there were. The argument used to pass from $\Phi$ to $\Phi_{0}$ in $\S 1$ shows that, if such a $\phi$ existed, there would be a nonzero entire $f$, also of exponential type $a$, but having only real 
zeros, such that

$$
\left|\frac{f(x)}{\Psi(x+i)}\right| \leqq 1
$$

say, for $-\infty<x<\infty$.

This, however, is impossible. We may, by a Phragmén-Lindelöf theorem ([3], p. 82), assume without loss of generality that $|\Psi(x+2 i)| \leqq 1$ for real $x$; then, by that same theorem,

$$
|\Psi(z+2 i)| \leqq \exp \{(\pi+\eta)|\Im z|\}
$$

Denote by $\mathfrak{N}\left(\rho, z_{0}\right)$ the number of zeros of $\Psi$ in the circle $\left|z-z_{0}\right| \leqq \rho$; Jensen's formula says that

$\log \left|\Psi\left(x_{p}+2 i\right)\right|=\frac{1}{2 \pi} \int_{0}^{2 \pi} \log \left|\Psi\left(x_{p}+2 i+r e^{i \vartheta}\right)\right| d \vartheta-\int_{0}^{r} \frac{\mathfrak{N}\left(\rho, x_{p}+2 i\right)}{\rho} d \rho$.

Because $\Psi(z)$ has a zero of order $\left[\Delta_{p}\right]$ at $x_{p}$, substitution of (16) into the last relation yields

$$
\log \left|\Psi\left(x_{p}+2 i\right)\right| \leqq\left(2+\frac{2 \eta}{\pi}\right) r-\left[\Delta_{p}\right] \log \frac{r}{2}, \quad r \geqq 2
$$

If $f$, of exponential type $a<\pi$, satisfies (15), then, since $\Psi(z)$ has only real zeros, the ratio $f(z) / \Psi(z+i)$ is of negative exponential

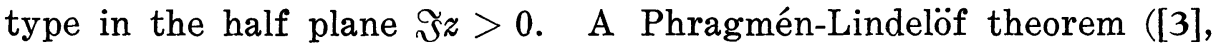
p. 82) now implies that

$$
\left|f\left(x_{p}+i\right)\right| \leqq\left|\Psi\left(x_{p}+2 i\right)\right|
$$

Calling $N\left(\rho, z_{0}\right)$ the number of zeros of $f(z)$ in the circle $\left|z-z_{0}\right| \leqq \rho$, Jensen's formula reads

(19) $\log \left|f\left(x_{p}+i\right)\right|=\frac{1}{2 \pi} \int_{0}^{2 \pi} \log \left|f\left(x_{p}+i+r e^{i \vartheta}\right)\right| d \vartheta-\int_{0}^{r} \frac{N\left(\rho, x_{p}+i\right)}{\rho} d \rho$.

Because the zeros of $f$ are real,

$$
\int_{0}^{r} \frac{N\left(\rho, x_{p}+i\right)}{\rho} d \rho \leqq N\left(r, x_{p}\right) \log r, \quad r \geqq 1
$$

We can represent $f(z)$ as a Hadamard product like the one on the right in (7), putting, without loss of generality, $\alpha=0$. From such a representation, it is easy to see that

$$
|f(z)| \geqq|f(\Re z)|
$$

Using this relation and the previous one in (19), one finds 


$$
\log \left|f\left(x_{p}+i\right)\right| \geqq \frac{1}{\pi} \int_{-r}^{r} \frac{\log \left|f\left(x_{p}+s\right)\right|}{\sqrt{r^{2}-s^{2}}} d s-N\left(r, x_{p}\right) \log r, \quad r \geqq 1,
$$

which, combined with (18) and (17), yields

$$
\begin{aligned}
\frac{1}{\pi} \int_{-r}^{r} \frac{\log \left|f\left(x_{p}+s\right)\right|}{\sqrt{r^{2}-s^{2}}} d s \leqq\left(2+\frac{2 \eta}{\pi}\right) r+\left[\Delta_{p}\right] \log 2 \\
\quad+N\left(r, x_{p}\right) \log r-\left[\Delta_{p}\right] \log r, \quad r \geqq 2 .
\end{aligned}
$$

This inequality will force $f$ to be identically zero. Fix a parameter $\lambda<1$, and, with $R_{p}=\Delta_{p} / 2$, integrate the $r$ in (20) over the range $\left[\lambda R_{p}, R_{p}\right]$, noting that, for large $p, \lambda R_{p}$ is $>2$ by (12). After this integration, the left side of (20) becomes

$$
\int_{-R_{p}}^{R_{p}} \log \left|f\left(x_{p}+s\right)\right| J\left(s / R_{p}\right) d s
$$

where

$$
\begin{aligned}
J\left(s / R_{p}\right) & =\frac{1}{\pi} \int_{\max \left(|s|, \lambda R_{p}\right)}^{R_{p}}\left(r^{2}-s^{2}\right)^{-1 / 2} d r \\
& =\frac{1}{\pi} \arg \cosh \left(R_{p} /|s|\right)-\frac{1}{\pi} \arg \cosh \max \left(\lambda R_{p} /|s|, 1\right)
\end{aligned}
$$

is bounded above by a positive constant $C_{\lambda}$ independent of $R_{p}$ for $0<|s| \leqq R_{p}$. (We may take $C_{\lambda}=(1 / \pi) \arg \cosh (1 / \lambda)$.) According to (15), there is no loss of generality in assuming that $|f(x)| \leqq 1$, $-\infty<x<\infty$, so, if $p$ is large, our integration of (20) yields

$$
\begin{aligned}
C_{\lambda} \int_{-R_{p}}^{R_{p}} & \log \left|f\left(x_{p}+s\right)\right| d s \leqq\left(1-\lambda^{2}\right)\left(\frac{1}{4}+\frac{\eta}{4 \pi}\right) \Delta_{p}^{2} \\
& +\frac{1-\lambda}{2} \Delta_{p}\left[\Delta_{p}\right] \log 2 \\
& +\frac{1-\lambda}{2}\left[N\left(\frac{\Delta_{p}}{2}, x_{p}\right) \log \left(\frac{\Delta_{p}}{2}\right)-\left[\Delta_{p}\right] \log \left(\frac{\lambda \Delta_{p}}{2}\right)\right] \Delta_{p} .
\end{aligned}
$$

One verifies that $\Delta_{p+1}>\Delta_{p}$, hence the intervals $\left[x_{p}-R_{p}, x_{p}+R_{p}\right]$ do not overlap. Since $R_{p}=o\left(x_{p}\right)$, it will clearly be enough to show, with the help of (21), that

$$
\sum_{p} \frac{1}{x_{p}^{2}} \int_{-R_{p}}^{R_{p}} \log \left|f\left(x_{p}+s\right)\right| d s=-\infty,
$$

for this will imply that

$$
\int_{-\infty}^{\infty} \frac{\log |f(t)|}{t^{2}+1} d t=-\infty
$$


making $f \equiv 0$ by a well known theorem ([3], p. 85).

To this end, observe that the right side of (21) is, for large $p$, bounded above by

$$
A \Delta_{p}^{2}+B\left\{N\left(\frac{\Delta_{p}}{2}, x_{p}\right)-\left[\Delta_{p}\right]\right\} \Delta_{p} \log \Delta_{p}
$$

with two positive constants $A$ and $B$, and that

$$
\sum_{8}^{\infty} \Delta_{p}^{2} / x_{p}^{2}<\infty
$$

by (12). We therefore need only establish

$$
\sum_{p} \frac{1}{x_{p}^{2}}\left\{N\left(\frac{\Delta_{p}}{2}, x_{p}\right)-\left[\Delta_{p}\right]\right\} \Delta_{p} \log \Delta_{p}=-\infty .
$$

Here, it is convenient to denote the number of zeros of $f(z)$ on the interval $[0, t]$ by $\nu(t)$, thus,

$$
N\left(\frac{\Delta_{p}}{2}, x_{p}\right)=\nu\left(x_{p}+\frac{1}{2} \Delta_{p}\right)-\nu\left(x_{p}-\frac{1}{2} \Delta_{p}\right) .
$$

Take a fixed parameter $\gamma>1$ and close to 1 (the exact manner of choosing $\gamma$ will be described later on), and put

$$
X_{m}=\gamma^{m}, \quad m=1,2,3, \cdots .
$$

We think of $\left\{X_{m}\right\}$ as a coarse sequence of points moving out towards $\infty$ along the real axis, and of $\left\{x_{p}\right\}$ as a finely distributed sequence of points interspersed among the $X_{m}$. Picking a very large $m$, let us estimate the sum

$$
\sum_{x_{m}<x_{p} \leqq X_{m+1}}\left\{\nu\left(x_{p}+\frac{1}{2} \Delta_{p}\right)-\nu\left(x_{p}-\frac{1}{2} \Delta_{p}\right)-\left[\Delta_{p}\right]\right\} \frac{\Delta_{p} \log \Delta_{p}}{x_{p}^{2}}
$$

from above.

Since $f(z)$ is of exponential type $a$, is bounded on the real axis, and has only real zeros, Levinson's density theorem ([10], pp. 33-41; [3], pp. 136-138; [9]) implies that

$$
\frac{\nu(t)}{t} \longrightarrow \frac{a}{\pi} \text { as } t \longrightarrow \infty,
$$

therefore, if $X^{\prime} \geqq \gamma X$ and $X$ is large enough, we have

$$
\nu\left(X^{\prime}\right)-\nu(X) \leqq\left(\frac{a}{\pi}+\varepsilon\right)\left(X^{\prime}-X\right)
$$

with an arbitrary $\varepsilon>0$. For large enough $m$, this yields, on account of (23), 


$$
\begin{gathered}
\sum_{x_{m}<x_{p} \leqq X_{m+1}}\left\{\nu\left(x_{p}+\frac{1}{2} \Delta_{p}\right)-\nu\left(x_{p}-\frac{1}{2} \Delta_{p}\right)\right\} \\
\leqq\left(\frac{a}{\pi}+2 \varepsilon\right)\left(X_{m+1}-X_{m}\right)
\end{gathered}
$$

because the intervals $\left[x_{p}-\Delta_{p} / 2, x_{p}+\Delta_{p} / 2\right]$ don't overlap and $\Delta_{p}=$ $o\left(x_{p}\right)$ by (12). According to (10) and (23),

$$
(m \log \gamma)^{3}<p \leqq((m+1) \log \gamma)^{3} \text { for } \quad X_{m}<x_{p} \leqq X_{m+1},
$$

so (12) gives, with an arbitrary $\varepsilon>0$,

$$
\frac{\Delta_{p} \log \Delta_{p}}{x_{p}^{2}} \leqq \frac{(1+\varepsilon) p^{-1 / 3}}{3 X_{m}} \leqq \frac{(1+\varepsilon)}{3 m X_{m} \log \gamma}
$$

if $X_{m}<x_{p} \leqq X_{m+1}$ and $m$ is large. Using this and (25), we find, by (23),

$$
\begin{gathered}
\sum_{x_{m}<x_{p} \leqq x_{m+1}}\left\{\nu\left(x_{p}+\frac{1}{2} \Delta_{p}\right)-\nu\left(x_{p}-\frac{1}{2} \Delta_{p}\right)\right\} \frac{\Delta_{p} \log \Delta_{p}}{x_{p}^{2}} \\
\leqq \frac{1}{3}\left(\frac{a}{\pi}+o(1)\right) \frac{\gamma-1}{\log \gamma} \cdot \frac{1}{m},
\end{gathered}
$$

where the $o(1)$ term tends to zero as $m \rightarrow \infty$.

When $m$ is large we obtain, on the other hand, from (23) and (12),

$$
\begin{aligned}
& \sum_{X_{m}<x_{p} \leqq x_{m+1}}\left[\Delta_{p}\right] \Delta_{p} \log \Delta_{p} / x_{p}^{2} \\
& \quad \geqq(1-o(1)) \sum_{(m \log r)^{3}<p \leqq((m+1) \log r)^{3}} 1 / 9 p=(1-o(1)) \cdot \frac{1}{9} \log \left(\frac{m+1}{m}\right)^{3} \\
& \quad=\frac{1}{3}(1-o(1)) \cdot \frac{1}{m}
\end{aligned}
$$

where, again, $o(1) \rightarrow 0$ as $m \rightarrow \infty$.

Substituting (26) and (27) into (24) we find that that expression is

$$
\leqq \frac{1}{3}\left[\frac{a}{\pi} \frac{\gamma-1}{\log \gamma}+o(1)-1\right] \cdot \frac{1}{m}
$$

if $m$ is large. The type, a, of $f$, was, however, supposed $<\pi$. We can therefore fix the parameter $\gamma$ used to define tht $X_{m}$ so close to 1 that

$$
\frac{a}{\pi} \frac{\gamma-1}{\log \gamma}<1
$$


and then, for all sufficiently large $m$, the sum in (24) will be

$$
\leqq-\frac{c}{m}
$$

with a positive constant $c$.

But this implies (22). Therefore, as was already stated, $f(z) \equiv 0$. Our assertion is proved.

3. Discussion. So, there we are. We have a multiplier theorem for weights $W(x)$ equal to entire functions of exponential type, but not for weights equal to the reciprocals of such functions, even though the functions' zeros stay away from the real axis.

One's first thoughts are that the methods used to prove Beurling and Malliavin's multiplier theorem, applying, as they do, to weights $W$ equal to functions of exponential type and satisfying (1), should also apply to weights $W(x)$ of the form $1 /|\Psi(x+i)|$ with functions $\Psi$ of exponential type bounded on the real axis and having all their zeros there. Our example shows, however, that this cannot be. What is going wrong?

The known proofs of the Beurling-Malliavin theorem ([2], [8], [11]) make use of two functionals involving $\log W$. One is the lefthand side of (1), and the other is quadratic in log $W$. During most of the remainder of this section, let us assume that $W(x) \geqq 1$ is even and that $W(0)=1$. Then, if

$$
W(x)=|F(x+i)|
$$

with an entire $F$ of exponential type having all its zeros on the real axis,

$$
\frac{\log W(x)}{x}=-\int_{0}^{\infty} \log \left|\frac{x+t}{x-t}\right| d\left(\frac{\nu(t)}{t}\right),
$$

where $\nu(t)=(1 / \pi)[\arg F(i)-\arg F(t+i)]$ is an increasing function of t. (See, for instance, [8], pp. 286-287.) As Beurling and Malliavin first observed ([11]), this formula represents $(\log W(x)) / x$ as a Green potential. The quadratic functional coming into play in proofs of the multiplier theorem is that Green potential's energy,

$$
\mathscr{E}\left(\frac{\log W(x)}{x}\right)=\int_{0}^{\infty} \int_{0}^{\infty} \log \left|\frac{x+t}{x-t}\right| d\left(\frac{\nu(t)}{t}\right) d\left(\frac{\nu(x)}{x}\right) .
$$

This energy is always nonnegative, and the proofs require that it be finite as well as fulfillment of (1).

In our present case, where $W(x)=|F(x+i)| \geqq 1$, the fact that $\nu(t)$ increases means that 


$$
\mathscr{E}\left(\frac{\log W(x)}{x}\right) \leqq \int_{0}^{\infty} \frac{\nu(x)}{x} \frac{\log W(x)}{x^{2}} d x
$$

But, $F$ being of exponential type, we have $\nu(x) / x \leqq$ const., so here finiteness of $\mathscr{E}(\log W(x) / x)$ follows from (1), because $|F(i)|=W(0)=$ 1 and the evenness of $W(x)$ make $\log W(x)=O\left(x^{2}\right)$ near 0 .

Suppose, however, that $W(x)=1 /|\Psi(x+i)|$ with an even $\Psi$ of exponential type having only real zeros such that $|\Psi(i)|=1$ and $|\Psi(x+i)| \leqq 1$ for $x$ real. Then,

$$
\frac{\log W(x)}{x}=\int_{0}^{\infty} \log \left|\frac{x+t}{x-t}\right| d\left(\frac{\nu(t)}{t}\right),
$$

where, here, $\nu(t)=(1 / \pi)[\arg \Psi(i)-\arg \Psi(t+i)]$ is again an increasing function of $t$. Relation (28) has been exactly turned around! The energy $\mathscr{E}((\log W(x)) / x)$ is again given by (29) but here, because of the change of sign, we have

$$
\mathscr{E}\left(\frac{\log W(x)}{x}\right) \leqq \int_{0}^{\infty} \frac{\log W(x)}{x^{2}} d \nu(x)
$$

with, in fact, both sides finite or infinite together, because their difference,

$$
\int_{0}^{\infty} \frac{\nu(x)}{x} \frac{\log W(x)}{x^{2}} d x
$$

is always finite in the present circumstance.

The difference between the two choices for $W(x)$, as $|F(x+i)|$ or as $1 /|\Psi(x+i)|$, is thus that in the first case the energy is bounded by a multiple of the left side of (1), while in the second it must be estimated by the right side of (31). Regarding the second case, where $W(x)=1 /|\Psi(x+i)|$, it turns out that if $\mathscr{E}((\log W(x)) / x)$ is finite, any of the proofs of the multiplier theorem can be pushed through, yielding the existence of nonzero entire $\varphi$ of arbitrarily small exponential type satisfying (3). Here, the right side of (31) equals

$$
\frac{1}{\pi} \int_{0}^{\infty} \frac{1}{x^{2}} \log \left|\frac{\Psi(i)}{\Psi(x+i)}\right|\left(\frac{\partial \log |\Psi(z+i)|}{\partial y}\right)_{z=x} d x,
$$

so, if this expression is finite, we have a multiplier theorem.

The example of $\S 2$ shows that the expression just written cannot be replaced by

$$
\int_{0}^{\infty} \frac{1}{x^{2}} \log \left|\frac{\Psi(i)}{\Psi(x+i)}\right| d x
$$

Some kind of regularity of $\log |\Psi(x+i)|$ is thus needed in addition 
to the property

$$
\left|\frac{d \log |\Psi(x+i)|}{d x}\right| \leqq \log \left|\frac{\text { const. }}{\Psi(x+i)}\right|
$$

which that function always has in the present circumstance (see the end of $\S 1$ ). It would be interesting to know exactly how much is needed.

That some kind of smoothness is involved seems indicated by another phenomenon. If $W(x) \geqq 1$ is any continuous weight tending to $\infty$ as $x \rightarrow \pm \infty$ and satisfying (1), the weighted exponentials

$$
e^{i \lambda x} / W(x) \text { with }-A \leqq \lambda \leqq A
$$

are certainly not dense in the space of continuous functions vanishing at $\pm \infty$ for any finite $A$. The ingenious extreme-point method of de Branges ([4], pp. 270-280) now provides us with an entire function $S(z)$ of exponential type exactly equal to $A$ (in both the upper and lower half planes), having only simple real zeros-call them $x_{n}$ - such that

$$
\sum_{n} \frac{W\left(x_{n}\right)}{\left|S^{\prime}\left(x_{n}\right)\right|} \leqq 1
$$

whilst

$$
\int_{-\infty}^{\infty} \frac{\log ^{+}|S(x)|}{1+x^{2}} d x<\infty
$$

For any $a>0$, the theorem on the multiplier furnishes, thanks to (33), a nonzero entire $\varphi$ of exponential type $\leqq a$, bounded on the real axis and satisfying $|S(x) \varphi(x)| \leqq 1$ there. Since the product is of exponential type $\leqq A+a$, by Bernstein's theorem ([3], pp. 206 and $210 \mathrm{ff}$ ),

$$
\left|\frac{d}{d x}(S(x) \varphi(x))\right| \leqq A+a, \quad-\infty<x<\infty .
$$

Putting $x=x_{n}$ and using (32), we see that

$$
\left|W\left(x_{n}\right) \varphi\left(x_{n}\right)\right| \leqq A+a
$$

for all $n$. Here, by Levinson's density theorem which was already applied in $\S 2$, the zeros $x_{n}$ of $S(z)$ have density $A / \pi$ on both the positive and negative real axes. That is, assuming the $x_{n}$ indexed according to their natural order on the real line,

$$
\lim _{n \rightarrow \infty} \frac{x_{n}}{n}=\lim _{n \rightarrow-\infty} \frac{x_{n}}{n}=\frac{\pi}{A} .
$$


This makes (34) seem close to the uniform bound

$$
|W(x) \varphi(x)| \leqq \text { const., }-\infty<x<\infty,
$$

especially since $A$ can be chosen very large to begin with. (Note, however, that the function $S(z)$, and hence the $\varphi$ of type $\leqq$ the given number $a$ will depend on $A$ !) The idea here is that if $W(x)$ is regular enough, (34) perhaps does imply a uniform bound on $W(x) \varphi(x)$. Again, exactly how much regularity would be needed to draw this conclusion is not clear.

The trouble is that de Brange's method does not give very precise information about the location of the points $x_{n}$. Essentially all that is known about them is that they have density $A / \pi$ in both the positive and negative directions.

4. An application. The construction of $\S 2$ can be used to obtain an example of a kind of quasi-analyticity.

We shall exhibit a sequence of real numbers $\lambda_{k}$, defined for $k= \pm 1, \pm 2, \pm 3, \cdots$, such that

(i) $\lambda_{-k}=-\lambda_{k}$ (symmetry)

(ii) the exponentials $e^{i \lambda_{k} t}$ are complete in $L_{2}(-A, A)$ if $A<\pi$

(iii) the exponentials $e^{i \lambda_{k} t}$ are not complete in $L_{2}(-A, A)$ if $A>\pi$

(iv) if $u(t)$, defined on $[0, \infty)$, is such that

$$
\int_{0}^{\infty}\left|u(t)-\sum_{k} A_{k}(n) e^{i \lambda_{k} t}\right|^{2} e^{-t} d t \underset{n}{\longrightarrow} 0
$$

for a sequence of finite sums $\sum_{k} A_{k}(n) e^{i \lambda_{k} t}$, and $u(t) \equiv 0$ a.e., on any interval $[0,2 h], h>0$, then $u(t) \equiv 0$ a.e., on $[0, \infty)$.

What is interesting here is our being able to do the construction with $\lambda_{k}$ having the symmetry property $(i)$. If symmetry were not required, we could just take the one-sided sequence $\lambda_{k}=k, k=1$, $2,3, \cdots$, and it would have properties (ii)-(iv).

Start with the sequence $\left\{x_{p}\right\}, p=8,9, \cdots$, used in $\S 2$, and take any small positive number $l$ less than $x_{8}$ and all the $\Delta_{p}=$ $x_{p}-x_{p-1}$ for $p>8$. On each of the intervals $\left[x_{p}-l, x_{p}\right]$, choose $\left[\Delta_{p}\right]$ different points; the collection of all these points, enumerated in increasing order, will be the one-sided sequence $\left\{\lambda_{k}\right\}, k=1,2, \cdots$. For $k<0$ we take $\lambda_{k}$ as $-\lambda_{-k}$. It is claimed that properties (ii)(iv) hold with these $\lambda_{k}$.

Let us here use $n(t)$ to denote the number of points $\lambda_{k}$ between 0 and $t$. As at the beginning of $\S 2$, we easily see that

$$
\frac{n(t)}{t} \longrightarrow 1 \text { as } t \longrightarrow \infty \text {, }
$$


and it is well known that this implies property (ii). (See, for instance, [10], p. 3; [3], p.235; [12], p.22.)

Just as at the beginning of $\S 2$, we verify that our present distribution function $n(t)$ satisfies (13). As in $\S 2$, we can obtain, for any $\eta>0$, an entire function of exponential type $\leqq \pi+\eta$, bounded on the real axis, whose zeros include all the points $\lambda_{k}, k= \pm 1, \pm 2$, $\pm 3, \cdots$. This is easily seen to imply property (iii) ([12], p. 26).

Property (iv) remains to be established. Suppose that $u(t)$ is identically zero on $[0,2 h]$ with $h>0$, and that (35) holds. It is convenient to write

$$
\begin{gathered}
v(t)=e^{-t / 2} u(t), \\
S_{n}(t)=e^{-t / 2} \sum_{k} A_{k}(n) e^{i \lambda_{k} t} ;
\end{gathered}
$$

then the Fourier transforms

$$
\hat{v}(\lambda)=\int_{0}^{\infty} e^{i \lambda t} v(t) d t
$$

and

$$
\widehat{S}_{n}(\lambda)=\int_{0}^{\infty} e^{i \lambda t} S_{n}(t) d t
$$

belong to the space $H_{2}$ (for the upper half plane), and (35) implies, by Plancherel's theorem, that

$$
\int_{-\infty}^{\infty}\left|\hat{v}(\lambda)-\hat{S}_{n}(\lambda)\right|^{2} d \lambda \underset{n}{\longrightarrow} 0 .
$$

Since $v(t) \equiv 0$ a.e., on $[0,2 h]$, we even have

$$
\widehat{v}(\lambda) \in e^{2 i n \lambda} H_{2} \text {. }
$$

From (37) we see by direct calculation that each function $\hat{S}_{n}(\lambda)$ is not only analytic for $\Im \lambda>0$ but also meromorphic in the whole complex plane, with its poles lying among the points $-\left(\lambda_{k}+i / 2\right)$.

Take now any number $\eta$ with $0<\eta<h$ and use the argument mentioned above to get a nonzero entire function $\Psi(\lambda)$ of exponential type $\leqq \pi+\eta$, bounded on the real axis and having only real zeros, among which figure all the points $\lambda_{k}, k= \pm 1, \pm 2, \pm 3, \cdots$.

I claim that

$$
g(\lambda)=e^{-i h \lambda} \hat{v}(\lambda) \Psi(\lambda+i / 2)
$$

is an entire function of exponential type $\leqq \pi+\eta-h<\pi$. For each $n$,

$$
g_{n}(\lambda)=e^{-i h \lambda} \widehat{S}_{n}(\lambda) \Psi(\lambda+i / 2)
$$


is equal to a finite sum

$$
\sum_{k} i A_{k}(n) e^{-i h \lambda} \frac{\Psi(\lambda+i / 2)}{\lambda+\lambda_{k}+i / 2}
$$

which is clearly entire and of exponential type, since $-\lambda_{k}=\lambda_{-k}$ is, for each $k$, a zero of $\Psi(\lambda)$. We see that, for each $n$,

$$
\left|g_{n}(\lambda)\right| \leqq C_{n} e^{h 3 \lambda} e^{(\pi+\eta)|\Im \lambda|} ;
$$

in fact, since $|\Psi(\lambda+i / 2)|$ is bounded for real $\lambda$, we have

$$
\int_{-\infty}^{\infty}\left|g_{n}(\lambda)\right|^{2} d \lambda \leqq \text { const . }
$$

by (38), and the $C_{n}$ in (42) can be taken independent of $n$ ([3], $\mathrm{p}$. 98). From (38) and (40) we now see that $g(\lambda)$ is also an entire function of exponential type, and, by (42),

$$
|g(\lambda)| \leqq C e^{(\pi+\eta-h)|\Im \lambda|} \quad \text { in } \quad \Im \lambda<0 .
$$

But the same holds for, say, $\Im \lambda \geqq 1$ by (39) and (40), since $\Psi$ is of exponential type $\leqq \pi+\eta$. So $g(\lambda)$ does have exponential type $<\pi$.

However, by (40),

$$
\frac{|g(\lambda+i / 2)|}{|\Psi(\lambda+i)|}=e^{h / 2}|\hat{v}(\lambda+i / 2)|,
$$

which is bounded for real $\lambda$ by (39). The reasoning of $\S 2$ now applies with almost no change to the function $\Psi$ introduced here, and, from (43), leads to the conclusion that $g(\lambda) \equiv 0$ because $g$ is of type $<\pi$. This makes $u(t) \equiv 0$ a.e., by (40) and (36), and property (iv) holds.

\section{REFERENCES}

1. A. Beurling and P. Malliavin, On the closure of characters and the zeros of entire functions, Acta Math., 118 (1967), 79-93.

2. — On Fourier transforms of measures with compact support, Acta Math., 107 (1962), 291-309.

3. R. Boas, Entire Functions, Academic Press, New York, 1954.

4. L. de Branges, Hilbert Spaces of Entire Functions, Prentice-Hall, Englewood Cliffs, 1968.

5. P. Koosis, Weighted quadratic means of Hilbert transforms, Duke Math. J., 38 (1971), 609-634.

6. - Moyennes quadratiques de transformées de Hilbert, et fonctions de type exponentiel, C. R. Acad. Sci. Paris, Sér. A, 276 (1973), 1201-1204.

7. — Sur la non-totalité de certaines suites d'exponentielles sur des intervalles assez longs, Ann. Ecole Norm. Supér. Paris, Sér 3, 75 (1958), 125-152.

8. - Harmonic estimation in certain slit regions and a theorem of Beurling and Malliavin, Acta Math., 142 (1979), 275-304. 
9. P. Koosis, Nouvelle démonstration d'un théorème de Levinson concernant la distribution des zéros d'une fonction de type exponentiel, Bull. Soc. Math. de France, 86 (1958), $27-40$.

10. N. Levinson, Gap and Density Theorems, Amer. Math. Soc. Colloq. Pubs XXVI, New York, 1940.

11. P. Malliavin, On the multiplier theorem for Fourier transforms of measures with compact support, Arkiv för Mat., 17 (1979), 69-81.

12. R. Redheffer, Completeness of sets of complex exponentials, Advances in Math., 24 (1977), 1-62.

Received March 10, 1980. The author was partially supported by NSF grant MCS 80-02955.

University of California

Los Angeles, CA 90024 



\section{PACIFIC JOURNAL OF MATHEMATICS}

\section{EDITORS}

DONALD BABBITT (Managing Editor)

University of Galifornia

Los Angeles, California 90024

Hugo RossI

University of Utah

Salt Lake City, UT 84112

C. C. MOORE AND ANDREW OGG

University of California

Berkeley, CA 94720
J. DugundJI

Department of Mathematics University of Southern California Los Angeles, California 90007

R. FinN and J. Milgram Stanford University Stanford, California 94305

\section{ASSOCIATE EDITORS}

R. ARENS

E. F. BeCKenbaCh

B. H. NEUManN

F. WOLF

K. YosHIDA

\section{SUPPORTING INSTITUTIONS}

UNIVERSITY OF ARIZONA

UNIVERSITY OF BRITISH COLUMBIA

CALIFORNIA INSTITUTE OF TECHNOLOGY

UNIVERSITY OF CALIFORNIA

MONTANA STATE UNIVERSITY

UNIVERSITY OF NEVADA, RENO

NEW MEXICO STATE UNIVERSITY

OREGON STATE UNIVERSITY
UNIVERSITY OF OREGON

UNIVERSITY OF SOUTHERN CALIFONIA

STANFORD UNIVERSITY

UNIVERSITY OF HAWAII

UNIVERSITY OF TOKYO

UNIVERSITY OF UTAH

WASHINGTON STATE UNIVERSITY

UNIVERSITY OF WASHINGTON 


\section{Pacific Journal of Mathematics}

\section{Vol. 95, No. $1 \quad$ September, 1981}

John Allen Beachy and William David Blair, On rings with bounded

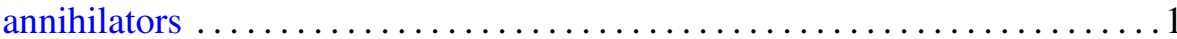

Douglas S. Bridges, A constructive look at positive linear functionals on

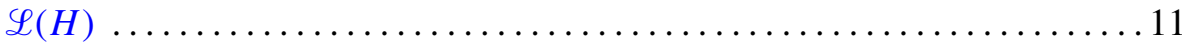

Muneo Chō and Makoto Takaguchi, Boundary points of joint numerical ranges

W. J. Cramer and William O. Ray, Solvability of nonlinear operator equations

Lester Eli Dubins and Gideon Schwarz, Equidiscontinuity of

Borsuk-Ulam functions

Maria Fragoulopoulou, Spaces of representations and enveloping 1.m.c.

*-algebras

Robert F. Geitz and J. Jerry Uhl, Jr., Vector-valued functions as families of scalar-valued functions

Ross Geoghegan, The homomorphism on fundamental group induced by a homotopy idempotent having essential fixed points

Ross Geoghegan, Splitting homotopy idempotents which have essential fixed points

Paul Jacob Koosis, Entire functions of exponential type as multipliers for

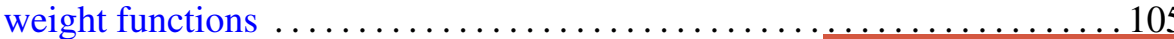

David London, Monotonicity of permanents of certain doubly stochastic matrices

Howard J. Marcum, Two results on cofibers

Giancarlo Mauceri, Zonal multipliers on the Heisenberg group

Edward Wilfred Odell, Jr. and Y. Sternfeld, A fixed point theorem in $c_{0} \quad \ldots 161$

Bernt Karsten Oksendal, Brownian motion and sets of harmonic measure zero

Andrew Douglas Pollington, The Hausdorff dimension of a set of normal numbers

Joe Repka, Base change lifting and Galois invariance ...

Gerald Suchan, Concerning the minimum of permanents on doubly stochastic circulants

Jun-ichi Tanaka, On isometries of Hardy spaces on compact abelian groups

Aaron R. Todd, Quasiregular, pseudocomplete, and Baire spaces 